OPEN ACCESS

Edited by:

Meihong Deng,

The Ohio State University,

United States

Reviewed by:

Ailing $L u$,

Feinstein Institute for Medical

Research, United States

Hai Huang,

The Ohio State University,

United States

*Correspondence:

Juan P. Cata

jcata@mdanderson.org

Wankun Chen

chenwank@163.com

Changhong Miao

miaochangh@163.com

${ }^{\dagger}$ These authors have contributed equally to this work and share first authorship

Specialty section:

This article was submitted to Microbes and Innate Immunity,

a section of the journal

Frontiers in Cellular and

Infection Microbiology

Received: 14 January 2021

Accepted: 25 February 2021

Published: 17 March 2021

Citation:

Chen Z, Zhang H, Qu M, Nan K, Cao H, Cata JP, Chen W and Miao C (2021) Review: The Emerging Role of Neutrophil Extracellular Traps in Sepsis and Sepsis-Associated Thrombosis. Front. Cell. Infect. Microbiol. 11:653228.

doi: 10.3389/fcimb.2021.653228

\section{Review: The Emerging Role of Neutrophil Extracellular Traps in Sepsis and Sepsis-Associated Thrombosis}

\author{
Zhaoyuan Chen ${ }^{1,2 \dagger}$, Hao Zhang ${ }^{1,2 \dagger}$, Mengdi Qu ${ }^{1,2}, \mathrm{Ke} \mathrm{Nan}^{1,2}$, Hanzhong $\mathrm{Cao}^{3}$, \\ Juan P. Cata ${ }^{4 *}$, Wankun Chen ${ }^{1,2,5 *}$ and Changhong Miao ${ }^{1,2 *}$
}

\begin{abstract}
${ }^{1}$ Department of Anesthesiology, Zhongshan Hospital, Fudan University, Shanghai, China, ${ }^{2}$ Cancer Center, Zhongshan Hospital, Fudan University, Shanghai, China, ${ }^{3}$ Anesthesiology and Surgical Oncology Research Group, Department of Anesthesiology and Perioperative Medicine, Nantong, China, ${ }^{4}$ Department of Anesthesiology and Perioperative Medicine, Anesthesiology and Surgical Oncology Research Group, The University of Texas MD Anderson Cancer Center, Houston, TX, United States, ${ }^{5}$ Zhangjiang Institute, Fudan University, Shanghai, China
\end{abstract}

Patients with sepsis commonly suffer from coagulation dysfunction and lead to the formation of thrombus. During the development of sepsis, neutrophils migrate from the circulating blood to infected tissues and mediate the formation of neutrophil extracellular traps (NETs) that kill pathogens. However, the overactivation of neutrophils can promote the formation of immunothrombosis and even cause disseminated intravascular coagulation (DIC), which damages microcirculation. The outcome of sepsis depends on early recognition and intervention, so clinical evaluation of NETs function may be a valuable biomarker for early diagnosis of sepsis. The interaction of NETs with platelets, complement, and endothelium mediates the formation of immunothrombosis in sepsis. Inhibiting the formation of NETs is also considered to be one of the potential treatments for sepsis. In this review, we will discuss the key role of neutrophils and NETs in sepsis and septic thrombosis, in order to reveal new mechanisms for thrombosis treatment of sepsis.

Keywords: sepsis, thrombosis, neutrophil extracellular traps, platelets, complement

\section{INTRODUCTION}

Sepsis is a life-threatening organ dysfunction caused by a disordered response to infection (Singer et al., 2016). In 2017, the incidence of global sepsis was estimated to be 48.9 million, of which the death toll related to sepsis was as high as 11 million (Rudd et al., 2020). In early sepsis, multiple pathophysiological processes exist simultaneously, such as inflammation and coagulation activation (Cecconi et al., 2018). The coagulation cascade is a complex process, and changes in patients' coagulation function during sepsis is a predictor of poor prognosis (Gotts and Matthay, 2016). The host will activate blood clotting in the process of defending against infectious pathogens. However, an excessive activation of the coagulation system along with vascular endothelial damage will cause the formation of microvascular thrombus and lead to multiorgan dysfunction (Gando, 2010). An hyperactivated and dysfunctional coagulation system will also manifest as consumption of anticoagulant factors, inhibition of the fibrinolytic system, significant shortening of clot 
formation kinetics (CFT), and increase clot firmness (CF) (Adamik et al., 2017). As a pro-inflammatory mediator, thrombin, a critical mediator of the coagulation cascade in sepsis, can activate receptors through specific proteases to stimulate monocytes and endothelial cells (ECs).

As the most abundant white blood cell group in humans, neutrophils play an essential role in the body's innate immunity caused by infection. An excessive activation of neutrophils can lead to the development of multiple organ dysfunction syndromes (Botha et al., 1995). In sepsis, pathogen-associated molecular patterns (PAMPs) or damage-associated molecular patterns (DAMPs) can activate pattern recognition receptors (PRRs) located in neutrophils (Kolaczkowska and Kubes, 2013). In these cells, those molecules will stimulate cell migration from circulating blood to infected tissues. Then, neutrophils will release intracellular granular components, including myeloperoxidase (MPO), neutrophil elastase (NE), and cathepsin G. Activated neutrophils will also release nuclear DNA and form a network structure containing nucleus, cytoplasm, and granular protein, also known as the neutrophil extracellular traps (NETs) (Brinkmann, 2004). NETs mediate the killing process of bacteria, viruses (Narasaraju et al., 2011; Saitoh et al., 2012) and fungi (Urban et al., 2006), however, their dysfunction will result in inability to fight infection, which is evidenced in patients with severe COVID-19 (Middleton et al., 2020). Excessive NETs formation can damage the microcirculation, promote immunothrombosis and lead to diffuse intravascular coagulation as they facilitate the formation of thrombus as a scaffold for thrombosis (Thomas et al., 2012).

In summary, NETs can eliminate pathogens by mediating neutrophils, but their hyperfunction will also cause tissue damage (Kaplan and Radic, 2012). In this review, we will discuss the key role of neutrophils and NETs in sepsis and sepsis thrombosis, to reveal new mechanisms for sepsis treatment.

\section{THE FORMATION OF NETS}

In 1971, Lerner first discovered the important role of neutrophils in the process of thrombosis (Lerner R. G. 1971). But it was not until 2004 when Brinkmann and others suggested the concept of NETs (Brinkmann, 2004).

Neutrophils can activate NADPH oxidase through protein kinase $\mathrm{C}(\mathrm{PKC})$ and Raf-MEK-ERK signaling pathways to produce reactive oxygen species (ROS). ROS-activated peptidylarginine deiminase 4 (PAD4) modifies specific arginine residues on histones $\mathrm{H} 3$ and $\mathrm{H} 4$ (Wang et al., 2004) to make linker histone $\mathrm{H} 1$ and heterochromatin protein $1 \mathrm{~b}$ dissociates from the nucleosome structure (Leshner et al., 2012), which mediates the dedensification of chromatin. MPO, NE, and cathepsin G promote further depolymerization of chromatin, thereby destroying the nuclear membrane (Clark et al., 2007). The chromatin is released into the cytoplasm as DAMP to further activate the immune response and form NETs. Treatment with DNase can significantly destroy the structure of NETs (Dąbrowska et al., 2016). The formation of NETs often leads to death of neutrophil, and this process is called NETosis (Vorobjeva and Chernyak, 2020). NETs can be formed in various parts of the body, and NETs can be released wherever neutrophils exist. Therefore, when sepsis occurs, neutrophils are present in different organs, such as the lung, liver, intestine, kidney, heart, and other organs, as well as in the blood circulation throughout the body. And the interaction of platelets, endothelial cells and complement can mediate the formation of NETs and immunothrombosis.

\section{MARKERS OF NETS}

The outcome of sepsis depends on early diagnosis and intervention. Thus, it has been suggested that early recognition of NETs may be a valuable biomarker of early sepsis. The degree of NETs formation is significantly related to the severity of the disease, and can independently predict the development of diffuse intravascular coagulation (DIC) and mortality (Abrams et al., 2019). Cell free DNA (cfDNA), NETs related MPO-DNA complex, citrullinated-histone H3-DNA (cit-H3-DNA) complex and deoxyribonuclease I (DNase I) can indirectly reflect NETs formation in the blood of patients with sepsis (Wang et al., 2016). For instance, cit-H3-DNA is also associated with NETs in patients with severe respiratory infections (Hirose et al., 2014). Also, DNA release, elastase production and inflammatory cytokines can affect the severity and organ failure of patients with sepsis (Kumar et al., 2019).

These markers have the potential to detect NETs, although they are not directly achieved by detecting NETs. At the same time, some markers are not only related to NETs, but also related to infections caused by other mechanisms. For example, the concentration of cfDNA in plasma can activate the nucleic acid sensing pathway to generate inflammation, and participate in the formation of sepsis (Sandquist and Wong, 2014). In addition, the circulating concentration of some markers is unstable, lacking detection sensitivity and specificity.

Abrams et al. (2019) found that co-incubation of neutrophils with the plasma or serum of patients with sepsis can directly induce the production of NETs. Compared with healthy volunteers, the plasma of patients with sepsis can cause a large number of NETs, and the degree of plasma NETs in patients with thrombocytopenia, abnormal prothrombin time, activated partial thromboplastin time, fibrinogen, and D-dimer are closely related. Therefore, these indicators can also be used as indirect means to reflex NETs (Abrams et al., 2019; Kumar et al., 2019). Correctly detecting the formation of NETs is essential for identifying early sepsis.

\section{FACTORS IN NETS-ASSOCIATED THROMBOSIS}

\section{Vascular Endothelium}

The integrity of endothelial structure and function is the key to maintaining vascular homeostasis. Endothelial injury plays an important role in thrombosis. In the development of sepsis, neutrophils are recruited to the site of inflammation through 
rolling, activation, firm attachment, and extravasation on ECs (Ley et al., 2007). The rolling of neutrophils on activated ECs is caused by the action of P-selectin and P-selectin glycoprotein ligand 1 (PSGL-1). By activating integrin $\alpha \mathrm{L} \beta 2$ (LFA-1) and binding to intercellular adhesion molecule 1 (ICAM-1), Pselectin participates in the further rolling and firm adhesion of neutrophils. Through the interaction between CXC chemokine ligand 1 (CXCL1) and its receptor CXC chemokine 2 (CXCR2), neutrophils are recruited to the site of inflammation (Grover and Mackman, 2018).

In septic-infected mice, the release of extracellular histones led to infiltration of neutrophils, vacuolation of ECs, intra-alveolar hemorrhage, and vascular thrombosis (Xu et al., 2009). When ICAM-1 inhibitors inhibited the homing of neutrophils to ECs, thrombosis in mice was reduced. NETs promote the activation of ECs and increase thrombosis through the synergistic effect of IL- $1 \alpha$ and cathepsin G (Folco et al., 2018). Matrix metalloproteinases-9 (MMPs-9) in NETs can activate MMP-2 of ECs and cause endothelial dysfunction (Carmona-Rivera et al., 2015). NETs can induce ECs to release adhesion factors and tissue factor (TF), further recruit inflammatory cells, and promote thrombosis (Rabinovitch, 2016). In sepsis, lipopolysaccharide (LPS) induces the activation of peptidylarginine deiminase $(\mathrm{PAD})$ and the formation of NETs mediated by the PAD-NET-CitH3 pathway, which changes the permeability of the pulmonary vascular endothelium (Liang et al., 2018). The induction of ECs by NETs also has two sides. Low concentrations of NETs can promote the release of inflammatory factors from ECs through the TLR4/NF- $\mathrm{KB}$ signaling pathway (Aldabbous et al., 2016), and NETs can also induce death of ECs in a dose-dependent manner (Saffarzadeh et al., 2012). NETs can amplify endothelial dysfunction associated with thrombosis. Vascular endothelium plays an important role in the occurrence and development of thrombus in sepsis.

\section{Platelets}

Platelets play an essential role in coagulation (van der Meijden and Heemskerk, 2019). Platelets can recruit leukocytes to the site of infection in the early stages of sepsis. Platelet activation induced by pathogens and platelet agonists, such as thrombin, arachidonic acid, collagen, or ADP can mediate the development of procoagulant, prothrombotic and inflammation, and ultimately lead to vascular endothelium damage. Activated platelets can also roll and adhere to the surface of neutrophils to form aggregates and enhance their ability to kill pathogens (Jenne et al., 2013; Jenne and Kubes, 2015). NETs further promote platelet adhesion, activation, aggregation, and exert procoagulant activity, thereby providing a scaffold for thrombosis (Fuchs et al., 2010), forming positive feedback activation of coagulation. NETs can also recruit red blood cells, promote von Willebrand Factor (VWF), fibrinogen and fibrin deposition, thereby inducing venous thrombosis (Fuchs et al., 2010).

Sepsis is usually accompanied by thrombocytopenia and occlusion of small blood vessels, called disseminated intravascular coagulation (DIC). The substances produced by NETs can either diffuse throughout the interstitium of organs, or be released into the vascular lumen and attach to the vascular wall of narrow capillaries (Carestia et al., 2016). In the mouse model of pneumonia caused by sepsis, thrombocytopenia usually worsened sepsis, led to increased bacterial growth in the blood and lungs, and reduced the survival rate of animals (de Stoppelaar et al., 2014).

LPS can initiate the non-classical activation of platelets, and activate TLR4 on platelets which will also lead to the release of the NETs (Carestia et al., 2016). The activation of platelets by LPS can induce platelet-dependent tissue factor pro-coagulant activity (TF-PCA) and promote the production of thrombin in a TLR4-dependent manner (Schattner, 2019). As one of the substances released by NETs, histones can induce the production of thrombin (Fuchs et al., 2011).

To a certain extent, NETs can trigger pro-thrombotic and pro-coagulant platelet-mediated responses by interacting with TLR4. As a key receptor, TLR4 on platelets may be an important factor in septic DIC (Semeraro et al., 2011). In septic mice, neutrophils adhere to the endothelial cells of the lung and liver sinusoids, and platelets activated by LPS can stick to neutrophils to promote the production of NETs (Clark et al., 2007). The $\alpha$ toxin produced by Staphylococcus aureus can bind to A disintegrin and metalloprotease 10 (ADAM10), which also causes the activation of platelets and neutrophils, and the formation of platelet-neutrophil aggregates (Powers et al., 2015). In addition, intravenous injection of $\alpha$-toxin can rapidly accumulate platelets and form thrombus in the liver sinusoids and glomeruli through GPIb, leading to multiple organ dysfunction (Surewaard et al., 2018). In COVID-19 patients, it was also found that the expression of P-selectin increased, and the aggregation of platelets-neutrophils and monocytes increased (Bhanu Kanth Manne, 2020) which was associated to thrombotic complications. Therefore, platelets play an important role in abnormal blood coagulation caused by sepsis (Figure 1).

\section{Complement}

In recent years, it has been discovered that NETs can activate three different complement pathways to form thrombosis. The complement system is stimulated by active coagulation factors, causing the immune system to recruit neutrophils (Berends et al., 2014). The complement system is composed of serine-protease cascade reactions, including the continuous cleavage of complement proteins, which ultimately leads to the formation of membrane attack complex (MAC) (Morgan, 2016).

Complement C3 is an important mediator in the complement system and activates neutrophils during sepsis (Yuen et al., 2016). Neutrophils from C3a receptor (C3aR) -deficient mice cannot form NETs. When C3 containing serum from healthy mice is exogenously administered, their function is restored. This shows that complement is closely related to the formation of NETs (Yipp et al., 2012). Blocking the complement receptor 3 (CR3) that binds to opsonin iC3b can inhibit NETosis (Behnen et al., 2014). C3b, as an important upstream component to generate MAC, has also been found in NETs (Behnen et al., 2014). On PMA-induced NETs, Factor B and C3 deposition have been found, and the combination of $\mathrm{Clq}$ and NETs can prevent itself from being degraded by DNase I (Papayannopoulos, 2018). 


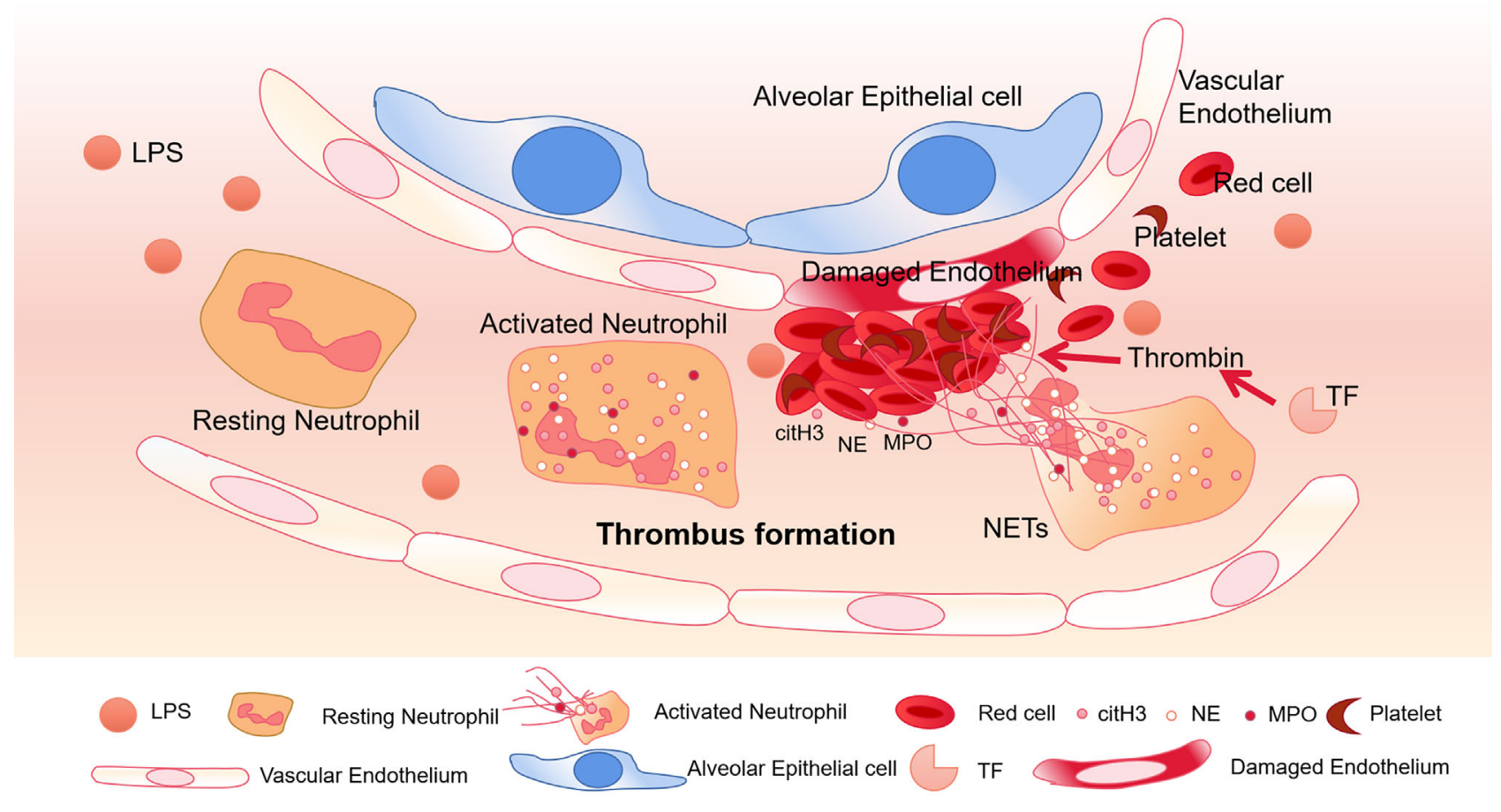

FIGURE 1 | Thrombus formation in platelets associated with NETs. In pneumonia of sepsis, LPS released by gram-negative bacteria can activate platelets. Neutrophils migrate into pulmonary capillaries, and interact with circulating platelets to form aggregates. Neutrophils release NETs, and citH3, NE, and MPO adhere to the NETs. Under the action of tissue factor (TF), NETs form thrombus together with red blood cells and platelets, destroying vascular ECs.

Thereby maintaining the structure of NETs (Elkon and Santer, 2012; Leffler et al., 2012). Complement C5a can recruit and activate neutrophils, leading to the up-regulation of immune receptors such as Toll-like receptors (TLR) and complement receptors (Wang et al., 2015). In addition, using C5a to prestimulate neutrophils enhanced their ability to generate NETs (Wang et al., 2015). The complement molecules in the blood can be deposited on NETs and continue to function. Properdin, Factor B and C3 have all been found to exist on NETs and exert their blood coagulation and antibacterial functions (Yousefi et al., 2009) (Figure 2).

\section{Immunothrombosis}

In recent years, researchers have proposed that there may be an effect mechanism in the link between blood coagulation and innate immune response that mediates the formation of immunothrombosis. Initially, NETs were found in patients' arterial or venous thrombosis. Researchers gradually realized that NETs provide a scaffold to recruit red blood cells, platelets, white blood cells, as well as bind plasma proteins. The aggregation of NETs in the coronary arteries suggested that NETs might contribute to the growth and stability of thrombus (de Boer et al., 2013). By analyzing ischemic stroke thrombosis, DNA, MPO and citrullinated histone $\mathrm{H} 4$ were found to be positive for triple costaining, and NETs were abundant in different types of stroke thrombosis (Ducroux et al., 2018). Infection accelerates neutrophils recruitment and activation, leading to a more pronounced involvement of NETs in thrombosis. In patients with abdominal aortic aneurysm infected with Porphyromonas gingivalis, the recruitment of neutrophils and the formation of NETs were increased, and wound healing was inhibited (Delbosc et al., 2011). Infection-driven NETs formation also leads to pulmonary thrombosis associated with sepsis (Jimenez-Alcazar et al., 2017). Because NETs can stimulate fiber remodeling, NETs may promote the development of venous thrombosis (Chrysanthopoulou et al., 2014). NETs may also be a prethrombotic lesion and play a key role in thrombosis.

The coagulation system is activated by pathogens and helps the host defend against infection by reducing the spread of pathogens and killing them (Engelmann and Massberg, 2013). Immunothrombosis can mediate the identification of pathogens and damaged cells, and inhibit the spread and survival of pathogens. This phenomenon also exists in sepsis. Neutrophils promote the capture and degradation of pathogens through the NETosis pathway, and the decondensed chromatin and antibacterial proteins are released from neutrophils to form NETs. Through the release of NETs, neutrophils participate in the formation of immunothrombosis. Intravascular coagulation induced by NETs resulted in extensive microvascular occlusion and multiple organ failure in a variety of sepsis mouse models (McDonald et al., 2017). 


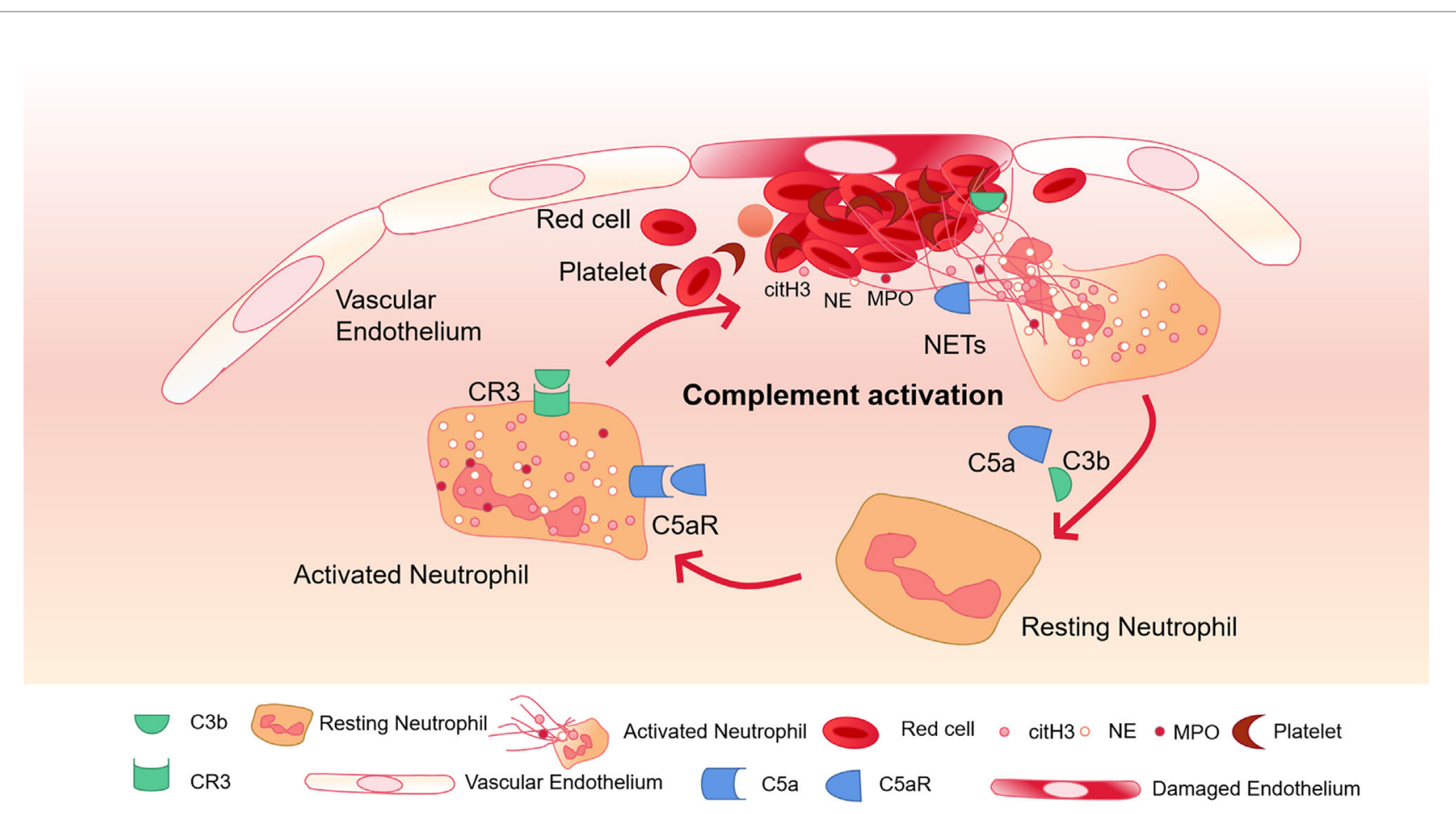

FIGURE 2 | Complement activation and NETs-mediated thrombosis. Complement C5a and C3b activate neutrophils and bind to CR3 and C5aR receptors on the surface of neutrophils to further promote the release of NETs. The activation of neutrophils can lead to endothelial cell damage, activation of the coagulation system, and further activation of complement through NETs, and finally amplify the inflammatory response.

The presence of senescent and pro-inflammatory neutrophils in the circulation of miR-146a-deficient mice leads to increased thrombosis in the LPS-induced sepsis model (Arroyo et al., 2020). Exposure of the endothelium to pathogens and PAMP triggers a pre-adhesion phenotype that leads to neutrophil binding. NF- $\kappa \mathrm{B}$ signal is involved in the circulatory process of thrombus and inflammation (Mussbacher et al., 2019). Neutrophils can secrete molecules that promote the initiation of the coagulation cascade (Darbousset et al., 2012), and TF plays an important role in this process (Smadja, 2014).

The two-way effect of NETs and platelets represents the connection between these phenomena. Whether in infectious or non-infectious diseases, the interaction of platelets and neutrophils is crucial (McDonald et al., 2017), and constitutes a vicious circle in the process of immunothrombosis Therefore, immunothrombosis may be an important physiological process of intravascular immunity, and its imbalance may be one of the potential events that trigger thrombotic diseases and lead to intravascular coagulation in sepsis.

\section{POTENTIAL THERAPEUTIC TARGETS}

Although NETs are increasingly recognized as important therapeutic targets, there is currently no clear drug that regulates
NETs to guide the treatment of sepsis and sepsis-related thrombosis. Several potential targets of NETs are listed below.

\section{TLR4 Blocker}

The downstream reaction caused by the combination of TLR4 and LPS is one of the classic pathways of sepsis. In addition, TLR4 on platelets is also involved in the induction of NETs in mice and humans (Clark et al., 2007). TLR $^{-1-}$ septic mice did not enhance the thrombotic response, and circulating ICAM-1 was significantly reduced (Obi et al., 2017). Inhibition of TLR4 can improve the prognosis of sepsis (Schattner, 2019), so TLR4 blockers may be used as a potential new method for the treatment of sepsis. 2-acetamidopyranoside compound (MW 389) acts as a TLR blocker to inhibit the production of TNF- $\alpha$ and iNOS RNA in a mouse model of LPS-induced sepsis (Neal et al., 2013). In addition, statins, and angiotensin receptor blockers (ARBs) have been found to have inhibitory activity on the TLR4 signaling pathway. Fluvastatin, simvastatin and atorvastatin showed effective inhibition of the TLR4 pathway (Gao et al., 2017). Candesartan can inhibit the activation of TLR4 induced by LPS (Dasu et al., 2009), and valsartan of the angiotensin-receptor blocker family (Yang et al., 2009) has been shown to reduce the release of pro-inflammatory cytokines by inhibiting TLR4 signaling. TAK-242 (resatorvid) is a small molecule specific inhibitor of TLR4. By binding to the intracellular domain of TLR4, it disrupts the interaction between 
TLR4 and adaptor molecules, thereby inhibiting TLR4 signal transduction and preventing the production of inflammatory mediators induced by LPS (Matsunaga et al., 2011). However, in large-scale clinical trials, TAK-242 failed to suppress cytokine levels in patients with sepsis, shock, or respiratory failure (Rice et al., 2010). Even if drugs are given in the early stages of sepsis, when sepsis is diagnosed, there is already an inflammatory response that cannot be suppressed. Therefore, it may be more important to recognize the signs of sepsis and prevent them in time.

As a ligand of LPS, TLR4 can prevent the cascade of inflammation by intervening in the early stage of sepsis, but its complete blockade may also lose the body's innate immune response to endotoxin. Therefore, it is still necessary to consider the pros and cons of its use before putting it into clinical use.

\section{PAD4 Inhibitors}

Overexpression of peptidyl arginine deiminase 4 (PAD4) leads to the elimination of chromatin and the release of NETs in cells in vitro. PAD4 causes the loss of the positive charge on the arginine guanidine group and unfolds the DNA strand to form NETs. PAD4 plays an important role in the formation of NETs (Franck et al., 2018). Therefore, the activation of PAD4 may be one of the targets of NETs formation. In a sepsis model, PAD4 knockout mice showed improved survival rate, reduced severity of organ dysfunction, and lower development of sepsis (Biron et al., 2018). PAD4 inhibitor BB-Cl-amidine can protect NETs-mediated vascular injury and endothelial dysfunction in mouse models (Knight et al., 2015). Perdomo et al. found that thrombocytopenia and thrombosis are two separable processes. Even in the absence of NETs, heparin may also lead to the reduction of platelets. Knockout of PAD4 or use of PAD4 inhibitor GSK484 will inhibit heparininduced thrombocytopenia/thrombosis (HIT) thrombocytopenia and thrombosis (Perdomo et al., 2019). PAD4 inhibitor GSK484 can also affect kindlin-3 in mouse neutrophils and inhibit thrombosis caused by NETs (Yan et al., 2019). The PAD4 inhibitor $\mathrm{Cl}$-amidine affects endothelial function and prethrombotic phenotype by blocking NETs (Knight et al., 2013), and can also inhibit tissue damage associated with inflammatory bowel disease mouse models (Chumanevich et al., 2011). Therefore, PAD4 may be one of the potential targets to inhibit the formation of NETs, which can interfere with thrombosis caused by inflammation without affecting the coagulation system.

\section{Heparin}

The role of heparin therapy in anticoagulation is unquestionable. However, the treatment of thrombus caused by heparin in NETs is still controversial. Low-dose heparin $(250 \mathrm{U} / \mathrm{kg})$ treatment can reduce the expression of NETs, histones, and pro-inflammatory factors (Sun et al., 2020). A large amount of heparin will produce antibodies against platelet factor 4 (PF4) and heparin complexes, leading to excessive activation of coagulation to activate platelets and promote thrombosis (Pouplard et al., 1999). In addition, heparin can directly induce the formation of NETs, but NETs cannot be induced by low molecular weight heparin, heparin analogs or heparin sulfate [low molecular weight heparin
(LMWH), heparin mimetics or heparan sulfate] (Lelliott et al., 2020). However, there are also reports in the literature that heparin and heparin-derived drugs (including unfractionated heparin, LMWH and occasionally fondaparinux) can induce immune responses, leading to HIT (Perdomo et al., 2019). This suggests that we need to pay attention to the dosage when using heparin in clinical practice, or use heparin substitutes for anticoagulation therapy to avoid activating NETs.

\section{Other Treatments}

In septic patients, the concentration of IL- 8 is closely related to the formation of NETs. IL-8 activation of mitogen-activated protein kinase is one of the main pathways for the formation of NETs in patients. Inhibition of IL-8 can significantly reduce NETosis (Abrams et al., 2019). Histones can activate neutrophils to form a network, and recombinant thrombomodulin (rTM) can inhibit the formation of NETs induced by histones (Binita Shrestha, 2019).

There is a dynamic platelet thrombin axis in sepsis, which promotes intravascular coagulation and microvascular dysfunction. DNase can inhibit the formation of NETs by degrading DNA. DNase1 and DNase1-like 3 can be expressed independently, degrade NETs in sterile neutrophilemia and sepsis, provide double protection, and protect the host from the harmful effects of intravascular NETs (Jimenez-Alcazar et al., 2017). Elimination of NETs by infusion of DNase, or in mice deficient in NETs formation caused by PAD4 deficiency, can be found to significantly reduce thrombin activity, platelet aggregation and improve microvascular perfusion (McDonald et al., 2017). A summary of potential targets for NETs therapy is shown in Table 1.

\section{CONCLUSION}

NETs have been confirmed to be related to inflammation, infection, and autoimmune diseases, and play an important

TABLE 1 | Potential targets for NETs therapy.

\begin{tabular}{|c|c|c|}
\hline Mechanism & Drug & References \\
\hline \multirow[t]{5}{*}{ TLR4 blocking agent } & MW 389 & (Neal et al., 2013) \\
\hline & $\begin{array}{l}\text { Statins (Fluvastatin, } \\
\text { Simvastatin, Atorvastatin) }\end{array}$ & (Gao et al., 2017) \\
\hline & ARBs (Candesartan, & (Dasu et al., 2009; \\
\hline & Valsartan) & Yang et al., 2009) \\
\hline & TAK-242 & $\begin{array}{l}\text { (Matsunaga et al., } \\
\text { 2011) }\end{array}$ \\
\hline \multirow[t]{3}{*}{ PAD4 inhibitor } & BB-Cl-amidine & (Knight et al., 2015) \\
\hline & GSK484 & (Perdomo et al., 2019) \\
\hline & Cl-amidine & (Knight et al., 2013) \\
\hline Heparin & Low-dose heparin & (Sun et al., 2020) \\
\hline Anti-IL-8 & Anti-IL-8 blocking mAb & (Abrams et al., 2019) \\
\hline $\begin{array}{l}\text { Prevent histone-induced } \\
\text { NETs formation }\end{array}$ & $\begin{array}{l}\text { Recombinant } \\
\text { thrombomodulin }\end{array}$ & (Binita Shrestha, 2019) \\
\hline \multirow[t]{2}{*}{ Degrade DNA in NETs } & DNase1 & $\begin{array}{l}\text { (Jimenez-Alcazar } \\
\text { et al., 2017) }\end{array}$ \\
\hline & DNase1-like 3 & $\begin{array}{l}\text { (Jimenez-Alcazar } \\
\text { et al., 2017) }\end{array}$ \\
\hline
\end{tabular}


role in sepsis and thrombosis caused by sepsis. NETs can not only play a role in immune defense in the inflammatory response, on the other hand, their excessive activation can also lead to a strong immune response and cause adverse consequences. In sepsis, NETs mainly through the interaction of neutrophils and platelets, leading to the functional damage of vascular ECs and the formation of immunothrombosis, further promoting the occurrence of microcirculation disorders.

The existence of NETs suggests that in the treatment of sepsis, whether a new method can be used to avoid excessive activation of inflammation and prevent the further occurrence of sepsis without affecting blood coagulation. By detecting the formation potential of NETs in the peripheral circulation, it can be used clinically to identify patients at risk of DIC and poor prognosis of sepsis. Based on the evidence that NETs and platelets interact to promote intravascular coagulation and its diffusion, we believe that the early recognition of NETs in sepsis can prompt early treatment of sepsis and DIC high-risk patients, and also provide a new direction for its treatment.

\section{REFERENCES}

Abrams, S. T., Morton, B., Alhamdi, Y., Alsabani, M., Lane, S., Welters, I. D., et al. (2019). A Novel Assay for Neutrophil Extracellular Trap Formation Independently Predicts Disseminated Intravascular Coagulation and Mortality in Critically Ill Patients. Am. J. Resp. Crit. Care 200 (7), 869-880. doi: 10.1164/rccm.201811-2111OC

Adamik, B., Gozdzik, W., Jakubczyk, D., Welna, M., and Kübler, A. (2017). Coagulation abnormalities identified by thromboelastometry in patients with severe sepsis. Blood Coagul. Fibrin. 28 (2), 163-170. doi: 10.1097/ MBC.0000000000000572

Aldabbous, L., Abdul-Salam, V., McKinnon, T., Duluc, L., Pepke-Zaba, J., Southwood, M., et al. (2016). Neutrophil Extracellular Traps Promote Angiogenesis: Evidence From Vascular Pathology in Pulmonary Hypertension. Arterioscler. Thromb. Vasc. Biol. 36 (10), 2078-2087. doi: 10.1161/ATVBAHA.116.307634

Arroyo, A. B., Fernández-Pérez, M. P., Monte, A. D., Águila, S., Méndez, R., Hernández-Antolín, R., et al. (2020). miR-146a is a pivotal regulator of neutrophil extracellular trap formation promoting thrombosis. Haematologica. doi: 10.3324/haematol.2019.240226

Behnen, M., Leschczyk, C., Moller, S., Batel, T., Klinger, M., Solbach, W., et al. (2014). Immobilized immune complexes induce neutrophil extracellular trap release by human neutrophil granulocytes via FcgammaRIIIB and Mac-1. J. Immunol. 193 (4), 1954-1965. doi: 10.4049/jimmunol.1400478

Berends, E. T., Kuipers, A., Ravesloot, M. M., Urbanus, R. T., and Rooijakkers, S. H. (2014). Bacteria under stress by complement and coagulation. Fems. Microbiol. Rev. 38 (6), 1146-1171. doi: 10.1111/1574-6976.12080

Bhanu Kanth Manne, F. D. E. A. (2020). Platelet gene expression and function in COVID-19 patients. Blood 136 (11), 1317-1329. doi: 10.1182/blood. 2020007214

Binita Shrestha, T. I. (2019). Recombinant Thrombomodulin Suppresses HistoneInduced Neutrophil Extracellular Trap Formation. Front. Immunol. 10, 2535. doi: 10.3389/fimmu.2019.02535

Biron, B. M., Chung, C. S., Chen, Y., Wilson, Z., Fallon, E. A., Reichner, J. S., et al. (2018). PAD4 Deficiency Leads to Decreased Organ Dysfunction and Improved Survival in a Dual Insult Model of Hemorrhagic Shock and Sepsis. J. Immunol. 123 (1), 33-42. doi: 10.1161/CIRCRESAHA.117.312494

Botha, A. J., Moore, F. A., Moore, E. E., Sauaia, A., Banerjee, A., and Peterson, V. M. (1995). Early neutrophil sequestration after injury: a pathogenic mechanism for multiple organ failure. J. Trauma. 39 (3), 411-417. doi: 10.1097/00005373199509000-00003

Brinkmann, V. (2004). Neutrophil Extracellular Traps Kill Bacteria. Science 303 (5663), 1532-1535. doi: 10.1126/science.1092385

\section{AUTHOR CONTRIBUTIONS}

$\mathrm{ZC}, \mathrm{HZ}, \mathrm{WC}$, and $\mathrm{CM}$ provided the conception and the structure of the article. ZC wrote the draft. All authors contributed to the article and approved the submitted version.

\section{FUNDING}

This research was supported by the National Key Research and Development Program of China (NO. 2020YFC2008400), the National Natural Science Foundation of China (NO.81873948, 81871591), Clinical Research Plan of SHDC (NO. SHDC 2020CR4064, SHDC2020CR1005A), Shanghai Shenkang hospital development center, clinical science and technology innovation project (NO. SHDC12018105), 2019 Fudan University Zhuo-Xue Project (NO. JIF159607); Shanghai Sailing Program (No. 20YF1418400); Shanghai Leading Talent (NO: 2019-112).

Carestia, A., Kaufman, T., and Schattner, M. (2016). Platelets: New Bricks in the Building of Neutrophil Extracellular Traps. Front. Immunol. 7, 271. doi: 10.3389/fimmu.2016.00271

Carmona-Rivera, C., Zhao, W., Yalavarthi, S., and Kaplan, M. J. (2015). Neutrophil extracellular traps induce endothelial dysfunction in systemic lupus erythematosus through the activation of matrix metalloproteinase-2. Ann. Rheumatol. Dis. 74 (7), 1417-1424. doi: 10.1136/annrheumdis-2013204837

Cecconi, M., Evans, L., Levy, M., and Rhodes, A. (2018). Sepsis and septic shock. Lancet 392, 10141,75-87. doi: 10.1016/S0140-6736(18)30696-2

Chrysanthopoulou, A., Mitroulis, I., Apostolidou, E., Arelaki, S., Mikroulis, D., Konstantinidis, T., et al. (2014). Neutrophil extracellular traps promote differentiation and function of fibroblasts. J. Pathol. 233, 294-307. doi: 10.1002/path.4359

Chumanevich, A. A., Causey, C. P., Knuckley, B. A., Jones, J. E., Poudyal, D., Chumanevich, A. P., et al. (2011). Suppression of colitis in mice by Cl-amidine: a novel peptidylarginine deiminase inhibitor. Am. J. Physiol. Gastrointest Liver Physiol. 300 (6), G929-G938. doi: 10.1152/ajpgi.00435.2010

Clark, S. R., Ma, A. C., Tavener, S. A., McDonald, B., Goodarzi, Z., Kelly, M. M., et al. (2007). Platelet TLR4 activates neutrophil extracellular traps to ensnare bacteria in septic blood. Nat. Med. 13 (4), 463-469. doi: 10.1038/nm1565

Dąbrowska, D., Jabłońska, E., Garley, M., Ratajczak-Wrona, W., and Iwaniuk, A. (2016). New Aspects of the Biology of Neutrophil Extracellular Traps. Scand. J. Immunol. 84 (6), 317-322. doi: 10.1111/sji.12494

Darbousset, R., Thomas, G. M., Mezouar, S., Frere, C., Bonier, R., Mackman, N., et al. (2012). Tissue factor-positive neutrophils bind to injured endothelial wall and initiate thrombus formation. Blood 120 (10), 2133-2143. doi: 10.1182/ blood-2012-06-437772

Dasu, M. R., Riosvelasco, A. C., and Jialal, I. (2009). Candesartan inhibits Toll-like receptor expression and activity both in vitro and in vivo. Atherosclerosis 202 (1), 76-83. doi: 10.1016/j.atherosclerosis.2008.04.010

de Boer, O. J., Li, X., Teeling, P., Mackaay, C., Ploegmakers, H. J., van der Loos, C. M., et al. (2013). Neutrophils, neutrophil extracellular traps and interleukin-17 associate with the organisation of thrombi in acute myocardial infarction. Thromb. Haemost. 109, 290-297. doi: 10.1160/TH12-06-0425

de Stoppelaar, S. F., van T, V. C., Claushuis, T. A., Albersen, B. J., Roelofs, J. J., and van der Poll, T. (2014). Thrombocytopenia impairs host defense in gramnegative pneumonia-derived sepsis in mice. Blood 124 (25), 3781-3790. doi: 10.1182/blood-2014-05-573915

Delbosc, S., Alsac, J. M., Journe, C., Louedec, L., Castier, Y., Bonnaure-Mallet, M., et al. (2011). Porphyromonas gingivalis participates in pathogenesis of human abdominal aortic aneurysm by neutrophil activation. Proof of concept in rats. PloS One 6, e18679. doi: 10.1371/journal.pone.0018679 
Ducroux, C., Di Meglio, L., Loyau, S., Delbosc, S., Boisseau, W., Deschildre, C., et al. (2018). Thrombus Neutrophil Extracellular Traps Content Impair tPAInduced Thrombolysis in Acute Ischemic Stroke. Stroke 49, 754-757. doi: 10.1161/STROKEAHA.117.019896

Elkon, K. B., and Santer, D. M. (2012). Complement, interferon and lupus. Curr. Opin. Immunol. 24 (6), 665-670. doi: 10.1016/j.coi.2012.08.004

Engelmann, B., and Massberg, S. (2013). Thrombosis as an intravascular effector of innate immunity. Nat. Rev. Immunol. 7 (2), e32366. doi: 10.1371/ journal.pone.0032366

Folco, E. J., Mawson, T. L., Vromman, A., Bernardes-Souza, B., Franck, G., Persson, O., et al. (2018). Neutrophil Extracellular Traps Induce Endothelial Cell Activation and Tissue Factor Production Through Interleukin-1alpha and Cathepsin G. Arterioscler. Thromb. Vasc. Biol. 38 (8), 1901-1912. doi: 10.1161/ ATVBAHA.118.311150

Franck, G., Mawson, T. L., Folco, E. J., Molinaro, R., Ruvkun, V., Engelbertsen, D., et al. (2018). Roles of PAD4 and NETosis in Experimental Atherosclerosis and Arterial Injury: Implications for Superficial Erosion. Circ. Res. 123, 33-42. doi: 10.1161/CIRCRESAHA.117.312494

Fuchs, T. A., Brill, A., Duerschmied, D., Schatzberg, D., Monestier, M., Myers, D. J., et al. (2010). Extracellular DNA traps promote thrombosis. Proc. Natl. Acad. Sci. U. S. A. 107 (36), 15880-15885. doi: 10.1073/pnas.1005743107

Fuchs, T. A., Bhandari, A. A., and Wagner, D. D. (2011). Histones induce rapid and profound thrombocytopenia in mice. Blood 118, 3708-3714. doi: 10.1182/ blood-2011-01-332676

Gando, S. (2010). Microvascular thrombosis and multiple organ dysfunction syndrome. Crit. Care Med. 38 (2 Suppl), S35-S42. doi: 10.1097/CCM. 0b013e3181c9e31d

Gao, W., Xiong, Y., Li, Q., and Yang, H. (2017). Inhibition of Toll-Like Receptor Signaling as a Promising Therapy for Inflammatory Diseases: A Journey from Molecular to Nano Therapeutics. Front. Physiol. 8, 508. doi: 10.3389/ fphys.2017.00508

Gotts, J. E., and Matthay, M. A. (2016). Sepsis: pathophysiology and clinical management. BMJ 353:i1585. doi: 10.1136/bmj.i1585

Grover, S. P., and Mackman, N. (2018). Neutrophils, NETs, and immunothrombosis. Blood 132 (13), 1360-1361. doi: 10.1182/blood-201808-868067

Hirose, T., Hamaguchi, S., Matsumoto, N., Irisawa, T., Seki, M., Tasaki, O., et al. (2014). Presence of neutrophil extracellular traps and citrullinated histone $\mathrm{H} 3$ in the bloodstream of critically ill patients. PloS One 9 (11), e111755. doi: 10.1371 /journal.pone. 0111755

Jenne, C. N., and Kubes, P. (2015). Platelets in inflammation and infection. Platelets 26 (4), 286-292. doi: 10.3109/09537104.2015.1010441

Jenne, C. N., Wong, C. H., Zemp, F. J., McDonald, B., Rahman, M. M., Forsyth, P. A., et al. (2013). Neutrophils recruited to sites of infection protect from virus challenge by releasing neutrophil extracellular traps. Cell Host Microbe 13 (2), 169-180. doi: 10.1016/j.chom.2013.01.005

Jimenez-Alcazar, M., Rangaswamy, C., Panda, R., Bitterling, J., Simsek, Y. J., Long, A. T., et al. (2017). Host DNases prevent vascular occlusion by neutrophil extracellular traps. Science 358, 1202-1206. doi: 10.1126/science.aam8897

Kaplan, M. J., and Radic, M. (2012). Neutrophil extracellular traps: double-edged swords of innate immunity. J. Immunol. 189 (6), 2689-2695. doi: 10.4049/ jimmunol.1201719

Knight, J. S., Zhao, W., Luo, W., Subramanian, V., O’Dell, A. A., Yalavarthi, S., et al. (2013). Peptidylarginine deiminase inhibition is immunomodulatory and vasculoprotective in murine lupus. J. Clin. Invest. 123 (7), 2981-2993. doi: 10.1172/JCI67390

Knight, J. S., Subramanian, V., O’Dell, A. A., Yalavarthi, S., Zhao, W., Smith, C. K., et al. (2015). Peptidylarginine deiminase inhibition disrupts NET formation and protects against kidney, skin and vascular disease in lupus-prone MRL/lpr mice. Ann. Rheumatol. Dis. 74 (12), 2199-2206. doi: 10.1136/annrheumdis2014-205365

Kolaczkowska, E., and Kubes, P. (2013). Neutrophil recruitment and function in health and inflammation. Nat. Rev. Immunol. 13 (3), 159-175. doi: 10.1038/ nri3399

Kumar, S., Gupta, E., Kaushik, S., Srivastava, V. K., Saxena, J., Mehta, S., et al. (2019). Quantification of NETs formation in neutrophil and its correlation with the severity of sepsis and organ dysfunction. Clin. Chim. Acta 495, 606610. doi: 10.1016/j.cca.2019.06.008
Leffler, J., Martin, M., Gullstrand, B., Tydén, H., Lood, C., Truedsson, L., et al. (2012). Neutrophil extracellular traps that are not degraded in systemic lupus erythematosus activate complement exacerbating the disease. J. Immunol. 188 (7), 3522-3531. doi: 10.4049/jimmunol.1102404

Lelliott, P. M., Momota, M., Shibahara, T., Lee, M. S. J., Smith, N. I., Ishii, K. J., et al. (2020). Heparin induces neutrophil elastase-dependent vital and lytic NET formation. Int. Immunol. 32 (5), 359-368. doi: 10.1093/intimm/dxz084

Lerner RG, G. R. C. G. (1971). Stimulation of human leukocyte thromboplastic activity by endotoxin. Proc. Soc Exp. Biol. Med. 138 (1), 145-148. doi: 10.3181/ 00379727-138-35848

Leshner, M., Wang, S., Lewis, C., Zheng, H., Chen, X. A., Santy, L., et al. (2012). PAD4 mediated histone hypercitrullination induces heterochromatin decondensation and chromatin unfolding to form neutrophil extracellular trap-like structures. Front. Immunol. 3, 307. doi: 10.3389/fimmu.2012.00307

Ley, K., Laudanna, C., Cybulsky, M. I., and Nourshargh, S. (2007). Getting to the site of inflammation: the leukocyte adhesion cascade updated. Nat. Rev. Immunol. 7 (9), 678-689. doi: 10.1038/nri2156

Liang, Y., Pan, B., Alam, H. B., Deng, Q., Wang, Y., Chen, E., et al. (2018). Inhibition of peptidylarginine deiminase alleviates LPS-induced pulmonary dysfunction and improves survival in a mouse model of lethal endotoxemia. Eur. J. Pharmacol. 833, 432-440. doi: 10.1016/j.ejphar.2018.07.005

Matsunaga, N., Tsuchimori, N., Matsumoto, T., and Ii, M. (2011). TAK242 (resatorvid), a small-molecule inhibitor of Toll-like receptor (TLR) 4 signaling, binds selectively to TLR4 and interferes with interactions between TLR4 and its adaptor molecules. Mol. Pharmacol. 79, 34-41. doi: 10.1124/ mol.110.068064

McDonald, B., Davis, R. P., Kim, S. J., Tse, M., Esmon, C. T., Kolaczkowska, E., et al. (2017). Platelets and neutrophil extracellular traps collaborate to promote intravascular coagulation during sepsis in mice. Blood 129 (10), 1357-1367. doi: 10.1182/blood-2016-09-741298

Middleton, E. A., He, X. Y., Denorme, F., Campbell, R. A., Ng, D., Salvatore, S. P., et al. (2020). Neutrophil extracellular traps contribute to immunothrombosis in COVID-19 acute respiratory distress syndrome. Blood 136 (10), 1169-1179. doi: 10.1182/blood.2020007008

Morgan, B. P. (2016). The membrane attack complex as an inflammatory trigger. Immunobiology 221 (6), 747-751. doi: 10.1016/j.imbio.2015.04.006

Mussbacher, M., Salzmann, M., Brostjan, C., Hoesel, B., Schoergenhofer, C., Datler, H., et al. (2019). Cell Type-Specific Roles of NF- $\mathrm{KB}$ Linking Inflammation and Thrombosis. Front. Immunol. 10, 85. doi: 10.3389/ fimmu.2019.00085

Narasaraju, T., Yang, E., Samy, R. P., Ng, H. H., Poh, W. P., Liew, A. A., et al. (2011). Excessive neutrophils and neutrophil extracellular traps contribute to acute lung injury of influenza pneumonitis. Am. J. Pathol. 179 (1), 199-210. doi: 10.1016/j.ajpath.2011.03.013

Neal, M. D., Jia, H., Eyer, B., Good, M., Guerriero, C. J., Sodhi, C. P., et al. (2013). Discovery and validation of a new class of small molecule Toll-like receptor 4 (TLR4) inhibitors. PloS One 8 (6), , e65779. doi: 10.1371/journal.pone.0065779

Obi, A. T., Andraska, E., Kanthi, Y., Kessinger, C. W., Elfline, M., Luke, C., et al. (2017). Endotoxaemia-augmented murine venous thrombosis is dependent on TLR-4 and ICAM-1, and potentiated by neutropenia. Thromb. Haemost. 117 (2), 339-348. doi: 10.1160/TH16-03-0218

Papayannopoulos, V. (2018). Neutrophil extracellular traps in immunity and disease. Nat. Rev. Immunol. 18 (2), 134-147. doi: 10.1038/nri.2017.105

Perdomo, J., Leung, H., Ahmadi, Z., Yan, F., Chong, J., Passam, F. H., et al. (2019). Neutrophil activation and NETosis are the major drivers of thrombosis in heparin-induced thrombocytopenia. Nat. Commun. 10 (1), 1322. doi: 10.1038/ s41467-019-09160-7

Pouplard, C., May, M. A., Iochmann, S., Amiral, J., Vissac, A. M., Marchand, M., et al. (1999). Antibodies to platelet factor 4-heparin after cardiopulmonary bypass in patients anticoagulated with unfractionated heparin or a lowmolecular-weight heparin : clinical implications for heparin-induced thrombocytopenia. Circulation 99 (19), 2530-2536. doi: 10.1161/01. cir.99.19.2530

Powers, M. E., Becker, R. E., Sailer, A., Turner, J. R., and Bubeck, W. J. (2015). Synergistic Action of Staphylococcus aureus alpha-Toxin on Platelets and Myeloid Lineage Cells Contributes to Lethal Sepsis. Cell Host Microbe 17 (6), 775-787. doi: 10.1016/j.chom.2015.05.011 
Rabinovitch, M. (2016). NETs Activate Pulmonary Arterial Endothelial Cells. Arterioscler. Thromb. Vasc. Biol. 36, 2035-2037. doi: 10.1161/ ATVBAHA.116.308206

Rice, T. W., Wheeler, A. P., Bernard, G. R., Vincent, J., Angus, D. C., Aikawa, N., et al. (2010). A randomized, double-blind, placebo-controlled trial of TAK-242 for the treatment of severe sepsis. Crit. Care Med. 38, 1685-1694. doi: 10.1097/ CCM.0b013e3181e7c5c9

Rudd, K. E., Johnson, S. C., Agesa, K. M., Shackelford, K. A., Tsoi, D., Kievlan, D. R., et al. (2020). Global, regional, and national sepsis incidence and mortality, 1990-2017: analysis for the Global Burden of Disease Study. Lancet 395, $200-$ 211. doi: 10.1016/S0140-6736(19)32989-7

Saffarzadeh, M., Juenemann, C., Queisser, M. A., Lochnit, G., Barreto, G., Galuska, S. P., et al. (2012). Neutrophil extracellular traps directly induce epithelial and endothelial cell death: a predominant role of histones. PloS One 7 (2), e32366. doi: 10.1371/journal.pone.0032366

Saitoh, T., Komano, J., Saitoh, Y., Misawa, T., Takahama, M., Kozaki, T., et al. (2012). Neutrophil extracellular traps mediate a host defense response to human immunodeficiency virus-1. Cell Host Microbe 12 (1), 109-116. doi: $10.1016 /$ j.chom.2012.05.015

Sandquist, M., and Wong, H. R. (2014). Biomarkers of sepsis and their potential value in diagnosis, prognosis and treatment. Expert. Rev. Clin. Immunol. 10 (10), 1349-1356. doi: 10.1586/1744666X.2014.949675

Schattner, M. (2019). Platelet TLR4 at the crossroads of thrombosis and the innate immune response. J. Leukocyte. Biol. 105 (5), 873-880. doi: 10.1002/ JLB.MR0618-213R

Semeraro, F., Ammollo, C. T., Morrissey, J. H., Dale, G. L., Friese, P., Esmon, N. L., et al. (2011). Extracellular histones promote thrombin generation through platelet-dependent mechanisms: involvement of platelet TLR2 and TLR4. Blood 118, 1952-1961. doi: 10.1182/blood-2011-03-343061

Singer, M., Deutschman, C. S., Seymour, C. W., Shankar-Hari, M., Annane, D., Bauer, M., et al. (2016). The Third International Consensus Definitions for Sepsis and Septic Shock (Sepsis-3). Jama 315, 801-810. doi: 10.1001/ jama.2016.0287

Smadja, D. M. (2014). Neutrophils as new conductors of vascular homeostasis. J. Thromb. Haemost. 12 (7), 1166-1169. doi: 10.1111/jth.12585

Sun, Y., Chen, C., Zhang, X., Wang, S., Zhu, R., Zhou, A., et al. (2020). Heparin improves alveolarization and vascular development in hyperoxia-induced bronchopulmonary dysplasia by inhibiting neutrophil extracellular traps. Biochem. Biophys. Res. Commun. 522 (1), 33-39. doi: 10.1016/j.bbrc.2019.11.041

Surewaard, B., Thanabalasuriar, A., Zeng, Z., Tkaczyk, C., Cohen, T. S., Bardoel, B. W., et al. (2018). alpha-Toxin Induces Platelet Aggregation and Liver Injury during Staphylococcus aureus Sepsis. Cell Host Microbe 24 (2), 271-284.e3. doi: 10.1016/j.chom.2018.06.017

Thomas, G. M., Carbo, C., Curtis, B. R., Martinod, K., Mazo, I. B., Schatzberg, D., et al. (2012). Extracellular DNA traps are associated with the pathogenesis of TRALI in humans and mice. Blood 119 (26), 6335-6343. doi: 10.1182/blood2012-01-405183

Urban, C. F., Reichard, U., Brinkmann, V., and Zychlinsky, A. (2006). Neutrophil extracellular traps capture and kill Candida albicans yeast and hyphal forms. Cell Microbiol. 8 (4), 668-676. doi: 10.1111/j.1462-5822.2005.00659.x van der Meijden, P., and Heemskerk, J. (2019). Platelet biology and functions: new concepts and clinical perspectives. Nat. Rev. Cardiol. 16 (3), 166-179. doi: 10.1038/s41569-018-0110-0

Vorobjeva, N. V., and Chernyak, B. V. (2020). NETosis: Molecular Mechanisms, Role in Physiology and Pathology. Biochem. (Mosc). 85 (10), 1178-1190. doi: $10.1134 /$ S0006297920100065

Wang, Y., Wysocka, J., Sayegh, J., Lee, Y. H., Perlin, J. R., Leonelli, L., et al. (2004). Human PAD4 regulates histone arginine methylation levels via demethylimination. Science 306 (5694), 279-283. doi: 10.1126/science.1101400

Wang, H., Wang, C., Zhao, M. H., and Chen, M. (2015). Neutrophil extracellular traps can activate alternative complement pathways. Clin. Exp. Immunol. 181 (3), 518-527. doi: 10.1111/cei.12654

Wang, H., Sha, L. L., Ma, T. T., Zhang, L. X., Chen, M., and Zhao, M. H. (2016), Circulating Level of Neutrophil Extracellular Traps Is Not a Useful Biomarker for Assessing Disease Activity in Antineutrophil Cytoplasmic AntibodyAssociated Vasculitis. PloS One 11 (2), e0148197. doi: 10.1371/ journal.pone.0148197

Xu, J., Zhang, X., Pelayo, R., Monestier, M., Ammollo, C. T., Semeraro, F., et al. (2009). Extracellular histones are major mediators of death in sepsis. Nat. Med. 15 (11), 1318-1321. doi: 10.1038/nm.2053

Yan, Y., Yang, H., Hu, X., Zhang, Z., Ge, S., Xu, Z., et al. (2019). Kindlin-3 in platelets and myeloid cells differentially regulates deep vein thrombosis in mice. Aging (Albany NY) 11 (17), 6951-6959. doi: 10.18632/aging.102229

Yang, J., Jiang, H., Yang, J., Ding, J. W., Chen, L. H., Li, S., et al. (2009). Valsartan preconditioning protects against myocardial ischemia-reperfusion injury through TLR4/NF-kappaB signaling pathway. Mol. Cell. Biochem. 330 (1-2), 39-46. doi: 10.1007/s11010-009-0098-1

Yipp, B. G., Petri, B., Salina, D., Jenne, C. N., Scott, B. N., Zbytnuik, L. D., et al. (2012). Infection-induced NETosis is a dynamic process involving neutrophil multitasking in vivo. Nat. Med. 18 (9), 1386-1393. doi: $10.1038 / \mathrm{nm} .2847$

Yousefi, S., Mihalache, C., Kozlowski, E., Schmid, I., and Simon, H. U. (2009). Viable neutrophils release mitochondrial DNA to form neutrophil extracellular traps. Cell Death Differ. 16 (11), 1438-1444. doi: 10.1038/cdd.2009.96

Yuen, J., Pluthero, F. G., Douda, D. N., Riedl, M., Cherry, A., Ulanova, M., et al. (2016). NETosing Neutrophils Activate Complement Both on Their Own NETs and Bacteria via Alternative and Non-alternative Pathways. Front. Immunol. 7, 137. doi: 10.3389/fimmu.2016.00137

Conflict of Interest: The authors declare that the research was conducted in the absence of any commercial or financial relationships that could be construed as a potential conflict of interest.

Copyright ( 2021 Chen, Zhang, Qu, Nan, Cao, Cata, Chen and Miao. This is an open-access article distributed under the terms of the Creative Commons Attribution License (CC BY). The use, distribution or reproduction in other forums is permitted, provided the original author(s) and the copyright owner(s) are credited and that the original publication in this journal is cited, in accordance with accepted academic practice. No use, distribution or reproduction is permitted which does not comply with these terms. 